\title{
Modelling traffic in detail with mesoscopic models: opening powerful new possibilities for traffic analyses
}

\author{
T. Vorraa ${ }^{1} \&$ A. Brignone ${ }^{2}$ \\ ${ }^{1}$ Citilabs Regional Director, Woking, Surrey, UK \\ ${ }^{2}$ Citilabs Regional Director, Cusano Milanino, Milan, Italy
}

\begin{abstract}
This paper discusses this 'new' model type and uses a new development in the Cube transport planning software package, Cube Avenue, to exemplify how mesoscopic dynamic traffic assignment models can help model very congested areas to the required level of detail.
\end{abstract}

Keywords: transportation planning, mesoscopic models, traffic congestion.

\section{Introduction}

Transport modelling is mostly done on a strategic level often categorised as 'macroscopic' modelling. For traffic engineering and area traffic control/intelligent traffic management purposes, the 'microscopic' models have become very popular and useful. The macroscopic models can cover a very large area but their shortfall for detailed traffic planning purposes is their inability to model the required level of detail in congested areas. The microscopic simulation models traffic dynamically and captures this level of detail perfectly, but its shortfall is the inability to model route choice properly and it is also very limited in terms of model sizes. So, there is scope for a 'mesoscopic' modelling level that can handle the right level of detail for large study areas.

Cube Avenue, an extension to the macroscopic planning module Cube Voyager, offers transportation professionals an innovative tool for analyzing traffic. With Cube Avenue, analysts can study problems for which traditional models don't provide enough data and for which microscopic models provide too much data. 
By explicitly modelling time, Cube Avenue offers an ideal environment for many studies, such as comparing policies for alleviating peak period congestion or examining the effectiveness of emergency evacuation plans. Indeed, pioneering transportation professionals can use Cube Avenue to meld planning with real-time operations, examining likely impacts of operational responses before implementation.

\section{How mesoscopic models differs}

Traditional transportation models, such as Cube Voyager's Highway program, use macroscopic techniques to study the flow of traffic from point to point. These models treat the entire volume of traffic travelling between an origin and destination as a single unit. The models compute the lowest-cost path for the traffic volume and compute congestion effects on a gross scale, using volumecapacity ratios and estimating resulting speeds.

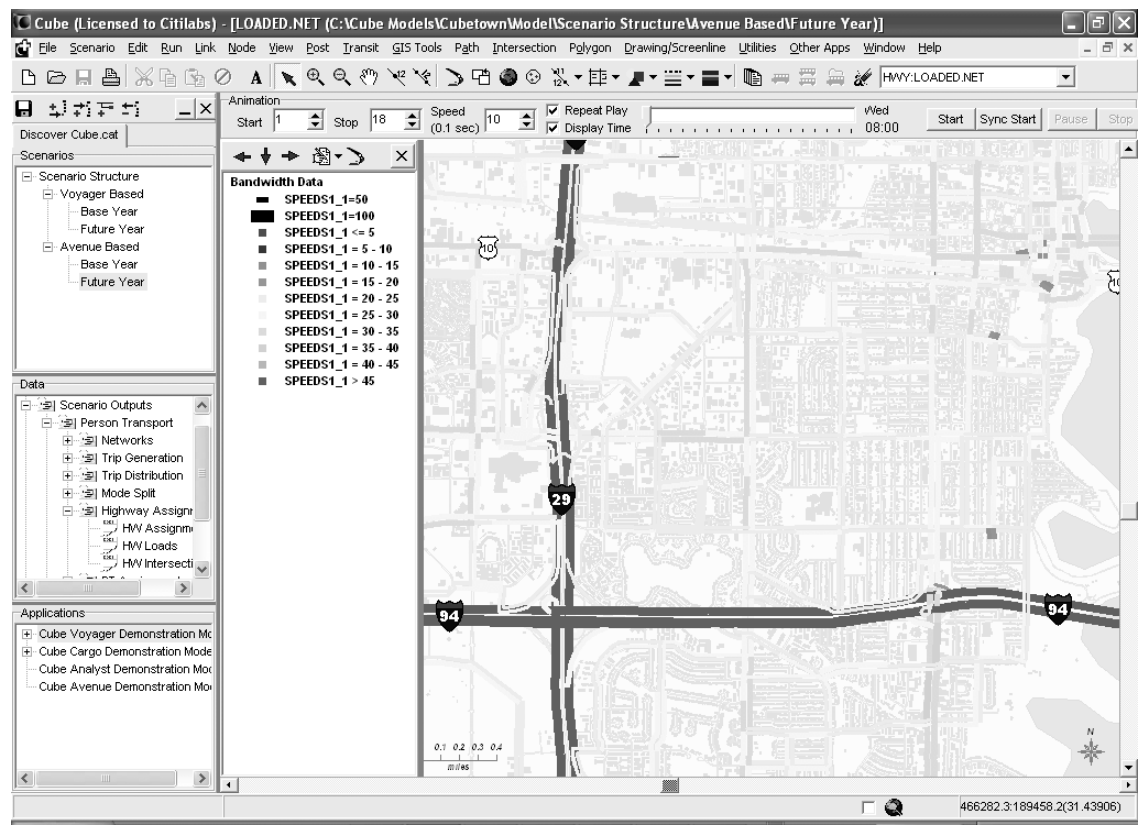

Figure 1: $\quad$ Predicted speed on all roads by time.

In contrast, the mesoscopic techniques can study traffic flow over time. In these models, the planner specifies the level of detail for vehicle, time, and network inputs. For example, one can examine traffic as individual vehicles or as platoons of multiple vehicles. Time is specified as segment of a few minutes or hours depending on the level of detail that is to be modelled. In detailed models intersection characteristics are usually specified. Using these inputs, the mesoscopic model in Cube Avenue computes the lowest-cost path for each 
vehicle unit, based on its departure time, and computes interactions among vehicle units as they travel through the network. Travel speeds are estimated based on vehicle density on road segments during each time increment. Because time is modelled explicitly, time-specific policies, such as variable road pricing or lane closures, can be studied using this technique.

Another Cube module, Cube Dynasim, uses microscopic techniques to present the most detail. This and other microsimulation tools model each vehicle explicitly and capture detailed movements and interactions, such as vehicle acceleration, lane changing and lane merges. Such detail makes microsimulation model the ideal tool for studying infrastructure geometry and traffic control systems or for studying multimodal projects, such as the interactions between pedestrian and vehicle flows. Of course, these detailed results require detailed inputs. The mesoscopic model on the other hand, produces details useful for many studies and requires less-detailed inputs - as an example, Cube Avenue requires about the same amount of data as a regional model.

\section{Uses of the mesoscopic model}

By considering new levels of detail, a mesoscopic model allows you to complete new types of analyses. With this approach, you can:

- Quantify impacts of upstream traffic congestion;

- Measure queuing at intersections and merge points in a network;

- Isolate secondary impacts from one intersection through another;

- $\quad$ Evaluate the benefits of ITS (intelligent transportation system) projects

- Simulate alternative infrastructure, operational, and policy changes to optimize;

- Emergency evacuation plans and strategies;

- $\quad$ Test strategies to improve arrival and departure from stadiums and other special event facilities.

\subsection{Use of Cube Avenue}

Cube Avenue is easy to use and works with traditional four-step transportation planning models or with any model type that uses highway assignment. You implement Cube Avenue with standard Cube Voyager commands and scripts. Therefore, current Cube Voyager users can easily implement a Cube Avenue model.

\subsection{Data inputs}

Most urban areas can use existing data in their regional transportation model to implement Cube Avenue. Cube Avenue inputs include:

- Roadway network in Cube Voyager format

- $\quad$ Peak period trip tables

- Vehicle storage area (vehicles per distance)

- Roadway distance, capacity, and lanes

- Traffic signal locations and characteristics 


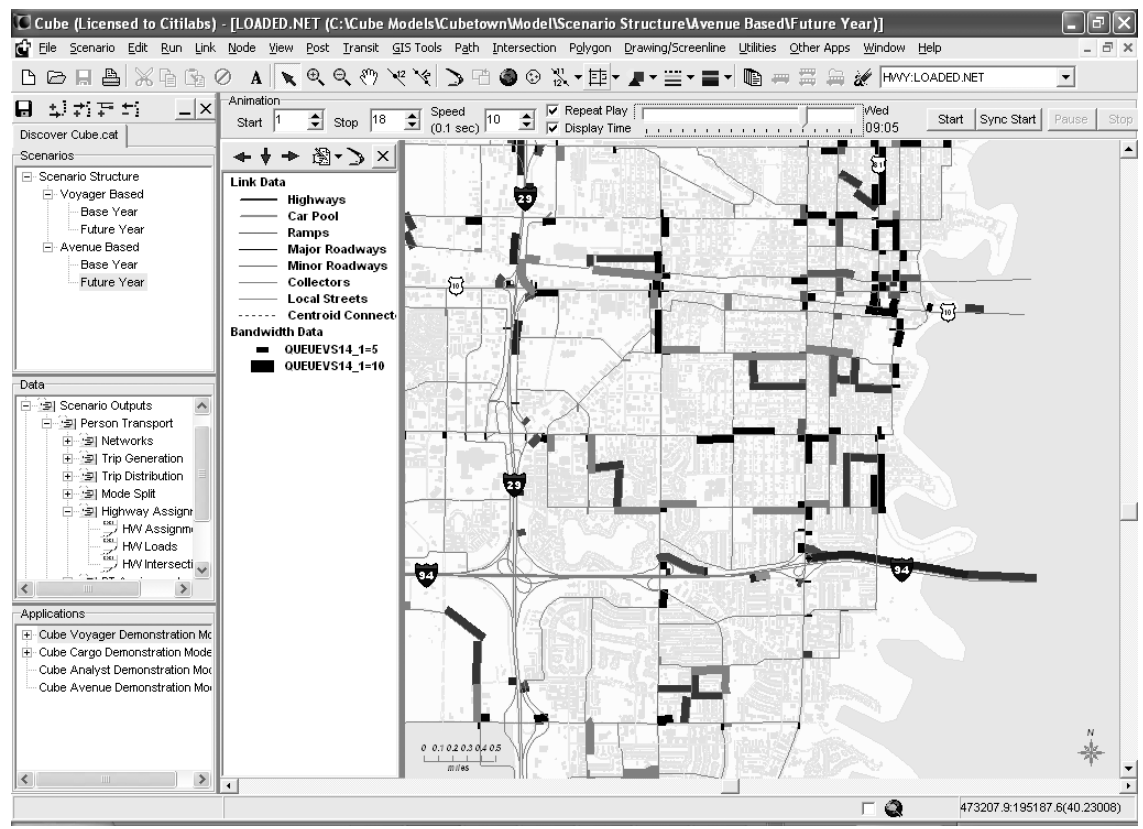

Figure 2: Isolate queues by length and time.

\section{Dynamic assignment process}

Cube Avenue is a dynamic equilibrium assignment model. Cube Avenue loads and tracks the movement of vehicle packets throughout the highway network. Vehicle packets can be any size - from an individual vehicle up to platoons of 20 or more vehicles. Cube Avenue explicitly simulates traffic flow and traffic control systems. It models traffic signals, roundabouts, stop-controlled intersections, and ramp merges. Vehicle packets move, stop, and queue through upstream roads and intersections. Through an iterative process, Cube Avenue calculates optimal network conditions. Typically, the model converges in several iterations, an extremely fast process for smaller systems and quite feasible for large urban areas. Indeed, modelers have successfully applied the system on areas with 3,000 zones and 25,000 links.

The dynamic assignment model is programmed using the Cube scripting language. To invoke the mesoscopic assignment algorithm, the path building and loading commands are replaced. Also, some additional parameters are required to define the time segments, queuing storage etc. An example script for the assignment procedure is given below. 
RUN PGM =HIGHWAY PRNFILE="C: \CUBE MODELS $\backslash$ APP 4ST \AAHWLO1A.PRN" MSG='HW DTA Assignment - using Cube Avenue'

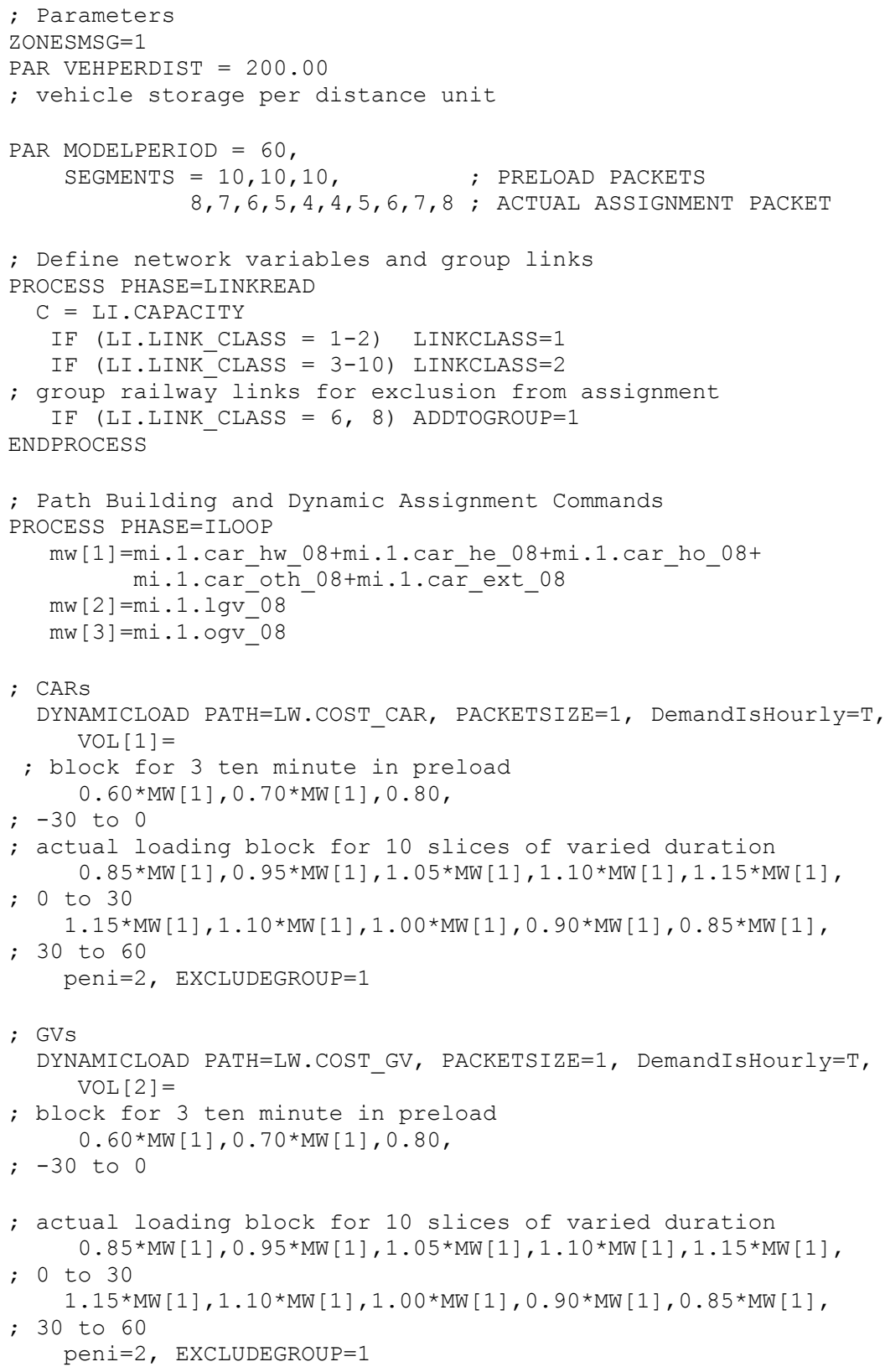

ENDPROCESS

ENDRUN 


\section{Performance measures}

Cube Avenue produces performance measures for time periods that you specify. For example, you can specify 15-minute results, one-hour results, or even oneminute results. Output performance indicators include:

- Total traffic volume on a road link

- Total traffic in queue

- $\quad$ Link operating speed and travel time

- Link occupancy/utilization

- Intersection LOS and operating conditions

When combined with Cube Base, Cube Avenue can help you analyze and visualize future operating conditions under various demands and road management strategies.

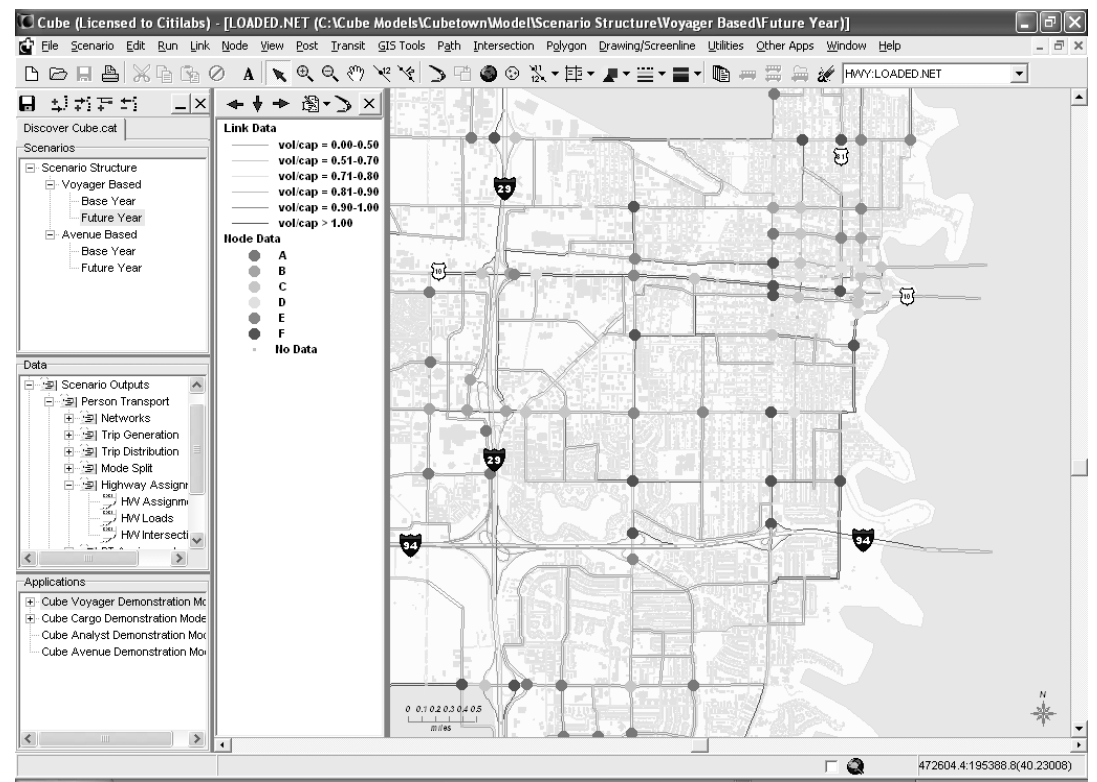

Figure 3: $\quad$ View Level of Service (LOS) at intersections.

\section{Examining the results}

Cube Avenue provides printed reports and data in standard Cube Voyager formats. You can view and animate the data files with Cube Base, and you can summarize the data with the charting and table functions in Cube Reports. Using standard Cube functions, you can easily compare alternatives. 


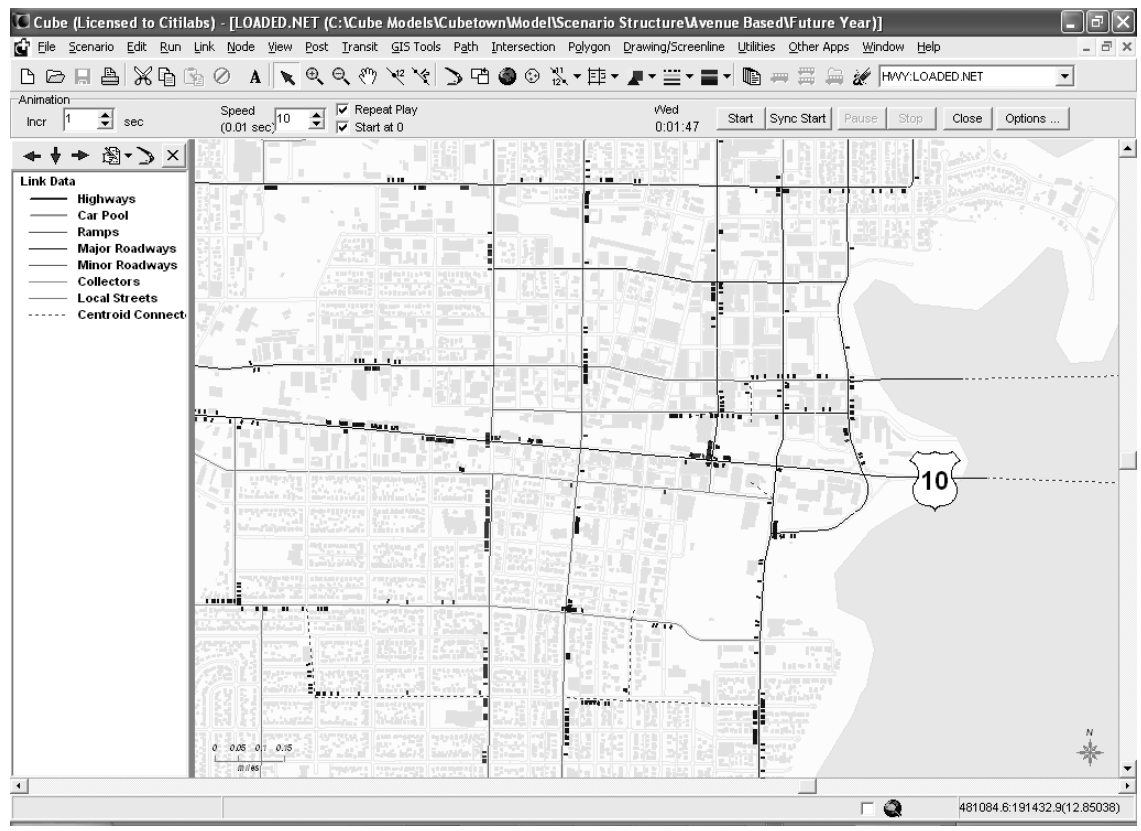

Figure 4: Animating the mesoscopic simulation - selected link flows in dark grey.

\section{First experiences with mesoscopic models in Cube Avenue}

The methodology described in this paper has been developed over the last couple of years and implemented in the Cube software framework. The first version of Cube Avenue was introduced to the Cube users early 2007 and there are several model development projects ongoing. There are currently ongoing model developments in the US, the UK, Scandinavia, Mainland Europe and Australia. Cube Avenue is currently used to examine evacuation plans, to study and optimise area traffic control, to examine blocking effects caused by tolling and general traffic management and planning issues.

\section{Conclusion}

Mesoscopic models can be used to accurately model congested urban areas. By splitting the model period into short time slices, the results will be much more realistic than traditional macroscopic models can achieve. The mesoscopic model offered in the Cube software package, Cube Avenue, can be bolted on to existing macroscopic demand models. 
666 Urban Transport XIV

\section{References}

[1] Roger Witte, Cube Avenue Theory, Cube Documentation System, Version 4.1.1, Citilabs December 2006.

[2] Ken Vaughn, Cube Avenue Example, Cube Demonstration Model, Version 4.1.1, Citilabs December 2006. 